\title{
INTROGRESIÓN DEL TAMAÑO DE LA GLUMA Y LA COBERTURA DEL GRANO EN POBLACIONES DE SORGO ${ }^{1}$
}

\author{
Leticia Alcalá-Salinas ${ }^{2}$, Francisco Zavala-García ${ }^{3}$, Noé Montes-García ${ }^{4}$, Sergio Castro-Nava ${ }^{5}$, \\ Omar G. Alvarado-Gómez ${ }^{6}$, Gilberto E. Salinas-García 6
}

\section{RESUMEN}

Introgresión del tamaño de la gluma y la cobertura del grano en poblaciones de sorgo. El objetivo de esta investigación fue determinar si la introgresión de líneas exóticas de sorgo incrementa la variabilidad del tamaño de la gluma y el porcentaje de grano descubierto. La población NESSC7 $\left(\mathrm{P}_{1}\right)$ se formó durante cuatro ciclos agrícolas (20052007), con un ciclo de introgresión, y a partir del quinto ciclo agrícola (2008) se estimó la variabilidad genética del caracter a través de 120 familias de medios hermanos y 110 familias de autohermanos. Se utilizó la metodología de Bloques Incompletos en dos localidades, Facultad de Agronomía de la Universidad Autónoma de Nuevo León (FAUANL) en Marín Nuevo León y el INIFAP en Río Bravo, Tamaulipas. La introgresión de líneas exóticas con menor tamaño de gluma y cobertura de grano en la población NESSC7 $\left(\mathrm{P}_{1}\right)$, mostró una disminución del tamaño de la gluma (13\%), un incremento del porcentaje de grano descubierto $(28 \%)$, y una mayor proporción de ambas características de la varianza aditiva (5\% y $1 \%$ ), heredabilidad en sentido estrecho de $22 \%$ y de $1 \%$ y mayor respuesta a la selección (4\% y $0,3 \%)$.

Palabras clave: Fitomejoramiento de Sorghum, varianza aditiva, heredabilidad en sentido estrecho.

\begin{abstract}
Introgression of glum size and grain cover into sorghum populations. The objective of the study was to determine if the introgression of exotic sorghum lines increases the variability in the size of the glum and in the percent of uncovered grains. The sorghum population NESSC7 $\left(\mathrm{P}_{1}\right)$ was formed during four agricultural cycles (2005-2007) with an introgression cycle, and beginning on the fifth agricultural cycle (2008), the genetic variability for these traits was estimated in the 120 half-silb and 110 selfsib families. The methodology of incomplete block design was used at two locations, the Faculty of Agronomy at the University of Nuevo Leon (FAUANL) in Marín Nuevo Leon and INIFAP in Río Bravo, Tamaulipas. The introgression of exotic lines with smaller glum size and reduced grain cover into the population NESSC7 $\left(\mathrm{P}_{1}\right)$ reduced the size of the $(13 \%)$, increased the percentage of uncovered grain (28\%), and increased the proportion of both characteristics in the additive variance $(5 \%$ and $1 \%$ ), the heritability in a narrow sense of $22 \%$ and $1 \%$ and higher response to selection (4\% y $0.3 \%)$.
\end{abstract}

Key words: Sorghum breeding, additive variance, narrow-sense heritability.

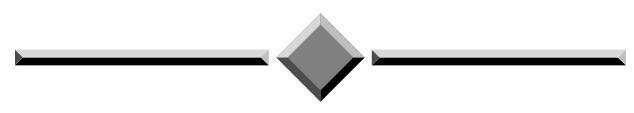

\footnotetext{
Recibido: 29 de mayo, 2009. Aceptado: 17 de mayo, 2010. Este trabajo corresponde a una Tesis de Doctorado realizada en la Facultad de Agronomía de la Universidad Autónoma de Nuevo León, México.

2 Estudiante de Doctorado del Programa de Producción Agrícola de la Facultad de Agronomía de la Universidad Autónoma de Nuevo León. Campus de Ciencias Agropecuarias, El Canadá, Gral. Escobedo, Nuevo León, México. Tel: 018113404399. leticia_alcala_s@hotmail.com

3 Facultad de Agronomía, Universidad Autónoma de Nuevo León, Escobedo, Nuevo León, México. f_zavala_g@hotmail.com

4 Instituto Nacional de Investigaciones Forestales, Agrícolas y Pecuarias (INIFAP)-Secretaría de Agricultura, Ganadería, Desarrollo Rural, Pesca y Alimentación (SAGARPA). Campo Experimental Río Bravo, Tamaulipas, México. nmontes62@hotmail.com

5 Unidad Académica Multidiciplinaria Agronomía y Ciencias (UAMAC), Universidad Autónoma de Nuevo León (UANL), Cd. Victoria, Tamaulipas, México. scastro@uat.edu.mx

6 Facultad de Agronomía de la Universidad Autónoma de Nuevo León (FAUANL). omar-alvarado@prodigy.net.mx; gisalina@hotmail.com
} 


\section{INTRODUCCIÓN}

La producción de grano de sorgo en México, se comercializa a través de centros de acopio o bodegas donde es almacenado. Durante este proceso se requiere de ciertos factores de calidad del grano dentro de los cuales, el porcentaje de restos de glumas son considerados como material diferente al grano y para la recepción de la cosecha del sorgo en las bodegas se considera como límite de contenido de impurezas del $1,5 \%$ a $4,0 \%$ (Secretaria de Comercio y Fomento Industrial 1994).

Las glumas forman parte de las inflorescencias del sorgo (S. bicolor), son hojas modificadas formadas por tejido fotosintético similar a las estructuras laminares (Everety 2000). En cada flor existen dos glumas que se encuentran sostenidas al pedicelo floral, éstas cubren los órganos florales, y generalmente se encuentran cerradas antes de la floración. Al iniciar la antésis se abren y permiten la emergencia de las anteras y estigmas, y después de la floración se cierran; las protuberancias del grano al desarrollarse las abren y deja cierto porcentaje del grano descubierto. En algunos tipos de sorgo las glumas son pequeñas o presentan un ángulo de abertura dejando un mayor porcentaje del grano descubierto. Pueden ser ovaladas, alternadas lanceoladas o contraídas lateralmente, tienen nervaduras o son lisas, pueden ser aristadas, mútica o mucronada (punta corta o aguda) (Clayton y Renvoize 1986).

Dependiendo de la apertura de las glumas queda un porcentaje descubierto del grano. La cobertura del grano se puede clasificar en: grano completamente cubierto, $25 \%$ descubierto, $50 \%$ descubierto, $75 \%$ descubierto y $100 \%$ descubierto (House 1985, ICRISAT 1999); sin embargo, por su tamaño, las glumas pueden ser más largas que el grano y mostrar segregación para el tamaño de gluma (USDA-ARS 2003). El ICRISAT (1999) midió el tamaño de ésta en 23400 accesiones, y encontró un tamaño de 2,4 a $2,8 \mathrm{~mm}$ en 9400 , de 2,9 a $3,4 \mathrm{~mm}$ en 9800 y de 3,5 a $3,9 \mathrm{~mm}$ en 4200 accesiones.

Durante la cosecha, los mecanismos de limpieza de las trilladoras separan las glumas del grano y las eliminan junto con la paja o residuos de cosecha, dejando los granos limpios que son depositados en el tanque de la trilladora, la cual los coloca en camiones para su transporte a las bodegas. En unos genotipos la gluma es larga, cubre el grano o se encuentra adherida a éste y dificulta las operaciones de limpieza del grano en la trilladora, fragmentos grandes de las glumas no logran atravesar los orificios del "saca-pajas" y quedan junto con los granos cosechados, provocando una reducción en la eficiencia de la trilladora y una cosecha de menor calidad debido al alto contenido de impurezas (material diferente al grano) (Chaparro 1997). Para mejorar la calidad de la cosecha del sorgo se pueden obtener genotipos con gluma pequeña, que facilite el desprendimiento y separación de la gluma del grano, aumente la eficiencia de los mecanismos de limpieza de la trilladora y reduzca el contenido de impurezas en los granos cosechados.

En el desarrollo de genotipos con menor tamaño de gluma y cobertura de grano, se puede hacer uso de la introgresión, con una población de apareamiento aleatorio aprovechando la esterilidad genética y líneas exóticas como fuente del carácter. El uso de plantas androestériles bajo apareamiento aleatorio permite la recombinación de genes que favorece la introducción del caracter de mejora (Shell y Becker 1986).

El gen que regula el caracter tamaño de la gluma en el sorgo (S. bicolor), $S g$ (gluma larga) domina a $s g$ (gluma pequeña) (Doggett 1970); sin embargo, pueden estar involucrados dos o tres genes con influencia citoplasmática (Bello y Obilana 1985, Khusnetdinova y El'konin 1989).

La introgresión se utiliza para introducir genes exóticos en poblaciones con el fin de incrementar la variabilidad y aumentar la frecuencia génica de un carácter deseado (Menkir et al. 1993, Ron y Hallauer 1997). Este método permite ampliar la base genética con genotipos adaptados que cuenten con un nuevo carácter de mejora y formar una población base sobre la cual se puede iniciar un proceso de selección para desarrollar líneas puras mejoradas (Menkir et al. 1993).

Es posible además ampliar la variabilidad genética del carácter tamaño de gluma y/o cobertura de grano en una población de sorgo con la introgresión. Esto se logra mediante nuevas recombinaciones con los genes favorables para gluma pequeña y/o menor cobertura de grano de las líneas exóticas; con ésto se puede obtener materiales genéticos que mejoren la eficiencia de la cosecha y proporcionen granos con menor contenido de impurezas.

A través de las familias de medios hermanos y autohermanos, se puede estudiar la estructura genética de una población, y se pueden estimar las medias y varianzas genéticas del carácter de interés; además de la heredabilidad y la respuesta predicha a la selección 
para determinar la proporción del caracter que se transmite a su progenie, al hacer selección para el carácter introducido (Falconer 1975, Márquez 1985).

Para las evaluaciones en el campo, cuando el número de familias es muy grande se puede utilizar el diseño experimental de bloques incompletos y análisis de varianza se realiza con base al método de diseños aumentados (augmented desings) (Federer 1956, Scott y Milliken 1993).

La varianza genética de acuerdo con Falconer (1975) resulta de la suma de las varianzas aditiva y de dominancia. La varianza aditiva se puede estimar a través de familias de medios hermanos, mientras que la dominancia se estima utilizando familias de autohermanos (Márquez 1985, Hallauer y Miranda 1988, Márquez y Sahagún 1994).

Los valores estimados de la varianza aditiva se utilizan para determinar la heredabilildad en sentido estrecho. Jan-orn et al. (1975) la estimaron en 196 familias de medios hermanos y 196 familias de autohermanos $\left(S_{1}\right)$ derivadas de una población y encontraron una heredabilidad en sentido estrecho de 0,82. Eckbil et al. (1977) determinaron una heredabilidad en familias de medios hermanos (MH) de 0,38 y Kenga et al. (2006) reportan una heredabilidad en sentido estrecho de 0,14 .

Las varianzas genéticas y heredabilidad en dos poblaciones derivadas de dos ciclos de selección recurrente de la población RP2B, fueron obtenidas por Mashark (2006) en progenies de medios hermanos (MH) y progenies $\mathrm{S}_{1}$ o familias de autohermanos (AH). Para rendimiento de grano se observó que en el primer ciclo, los AH obtuvieron valores más altos que los $\mathrm{MH}$ y que en el tercer ciclo de selección recurrente, por efecto de las autofecundaciones, los $\mathrm{AH}$ redujeron su rendimiento un $23,8 \%$ con respecto a los $\mathrm{MH}$. Reportan así mismo valores de heredabilidad para el rendimiento de 0,60 en los MH y 0,67 en los $\mathrm{AH}$.

En un estudio de la variabilidad genética del rendimiento de grano en 30 poblaciones de sorgo (Bello et al. 2007), se estimó una media del rendimiento de 47,0 g, una varianza genética de 394961 y una heredabilidad en sentido estrecho de 0,10 .

La precisión de las estimaciones de las varianzas genéticas se pueden determinar mediante el coeficiente de variación genética $(\mathrm{CVG})$, por lo tanto, un valor alto del CVG indica mayor varianza genética (Kenapp et al. 1987, Moreno et al. 2002 y Bello et al. 2007).
El objetivo del presente trabajo fue determinar si la introgresión de líneas exóticas de sorgo incrementa la variabilidad del tamaño de la gluma y del porcentaje de grano descubierto.

\section{MATERIALES Y MÉTODOS}

Este estudio se realizó en la Facultad de Agronomía de la Universidad Autónoma de Nuevo León (FAUANL), en Marín, Nuevo León, su ubicación geográfica es $25^{\circ} 52^{\prime} 13,5^{\prime \prime}$ L. N. y $100^{\circ} 02^{\prime} 22,56^{\prime}$ " L. O. a una altura de $355 \mathrm{msnm}$; y en el campo experimental del Instituto Nacional de Investigaciones Forestales, Agrícolas y Pecuarias (INIFAP) en Río Bravo, Tamaulipas localizado entre las coordenadas geográficas $25^{\circ} 57^{\prime} 53^{\prime \prime} \mathrm{L}$. N y $98^{\circ} 1^{\prime}$ 6" L. O. a una altura de $23 \mathrm{msnm}$. La precipitación anual registrada para el año 2008 en la localidad de Marín fue de 595 $\mathrm{mm}$ y en la localidad de Río Bravo fue de $640 \mathrm{~mm}$. Se utilizó la población de sorgo NESSC7 desarrollada por la Universidad de Nebraska seleccionada para tamaño de semilla grande y mayor calidad de grano; también de esta Universidad, se utilizaron seis líneas exóticas (NAGA-WITHE, MACIA, N559R, IR204, N570R y N571R), de la Universidad de Texas A \& M se utilizaron siete líneas exóticas (SC 301, SC 27914E, 88 BE2668, SC 1337, SC 1339, SC1341 y SC 1342) y una línea del ICRISAT (IS 4647). La población se utilizó como fuente del gen $\mathrm{ms}_{3}$ que genera plantas androestériles. Las 14 líneas exóticas se utilizaron como fuentes del carácter tamaño de gluma y/o cobertura de grano.

En el primer ciclo agrícola, otoño 2005 (julio a diciembre), se estimó el tamaño de la gluma y la cobertura de grano de las 14 líneas exóticas, de las cuales se seleccionaron las líneas con el menor tamaño de gluma y menor cobertura de grano; también en este ciclo se realizaron los primeros cruzamientos de las líneas exóticas seleccionadas con las plantas androestériles de la población.

Para estimar el tamaño de gluma y/o cobertura de grano se escogieron cinco panojas de cada línea exótica; de cada panoja se seleccionaron tres granos con gluma (en total se midieron 15 muestras por línea exótica). Antes de medir la gluma, se observó en el esteroscopio el grano con gluma y se estimó su cobertura y el porcentaje de grano descubierto (\% GD) con base 
a la clasificación de House (1985), ICRISAT (1999) y USDA-ARS (2003). Después se quitó la gluma y se midió su longitud en mm con el micrómetro ocular, para estimar su tamaño.

En el segundo ciclo agrícola, primavera 2006 (febrero a junio), la semilla $F_{1}$ de cada uno de los diferentes tipos de cruzamiento, se sembraron panoja por surco en cuatro surcos de cinco metros de largo, con una distancia entre ellos de $0,80 \mathrm{~cm}$ y 12 plantas por metro lineal; posteriormente, en la etapa de floración se autofecundaron 10 plantas de cada tipo de cruza. Se cubrió la inflorescencia con bolsas de papel; cada panoja se cosechó por separado y la semilla se guardó en sobres individuales (generación $\mathrm{F}_{2}$ de la primera cruza) con el propósito de encontrar plantas androestériles y poder realizar la recombinación apropiada.

En el tercer ciclo, otoño 2006 (julio a diciembre), se elaboró una mezcla de semilla en proporciones similares de cada panoja cosechada en el ciclo anterior de las plantas $\mathrm{F}_{2}$ de todos los cruzamientos, para sembrar un lote aislado de 22 surcos de 40 metros. Las plantas androestériles se identificaron y se dejaron a libre apareamiento para favorecer la recombinación de genes de la población y de las líneas exóticas, utilizadas para la introgresión de gluma pequeña y/o grano descubierto (Shell y Becker 1986); solo se cosecharon las panojas de las plantas androestériles y la semilla se guardó en sobres individuales.

En el cuarto ciclo, otoño 2007 (julio a diciembre), se sembró un lote de 88 surcos de cinco metros de largo, 44 surcos de la población NESSC7 $\left(\mathrm{P}_{0}\right)$ y 44 surcos de la población NESSC7 $\left(\mathrm{P}_{1}\right)$. Estos lotes se utilizaron para la formación de las familias de $\mathrm{MH}$ y de $\mathrm{AH}$ a través de las cuales se hicieron las estimaciones de las varianzas genéticas. Para formar las familias $\mathrm{MH}$ en las poblaciones original $\left(\mathrm{P}_{0}\right)$ y con un ciclo de introgresión $\left(\mathrm{P}_{1}\right)$, se identificaron plantas androestériles al iniciar la floración y se taparon con bolsa de papel; cuando la panoja completó la floración fueron polinizadas utilizando una mezcla de polen de 10 plantas fértiles tomadas al azar de la misma población, las panojas se cosecharon en forma individual; la semilla cosechada de cada planta correspondió a una familia de medios hermanos (Márquez y Sahagún 1994). Para la formación de las familias de AH se identificaron al azar plantas fértiles y se taparon con bolsa de papel; cada panoja formó una familia de AH (Márquez 1997).

En el quinto ciclo, primavera 2008 (febrero a junio), en dos localidades de la región noreste de México
(Marín, Nuevo León y Río Bravo, Tamaulipas) se empleó un diseño experimental de bloques incompletos. En Marín se sembraron en el campo experimental de la FAUANL el día 11 de marzo del 2008, 120 familias de MH y 110 familias de la población AH NESSC7 $\left(\mathrm{P}_{1}\right)$ y la misma cantidad de familias de la población NESSC7 $\left(\mathrm{P}_{0}\right)$. En la localidad Río Bravo, en el campo experimental del INIFAP el experimento se sembró el día 7 de marzo del 2008 con igual cantidad de familias de las dos poblaciones que se utilizaron en Marín.

Las familias de la población NESSC7 $\left(\mathrm{P}_{1}\right)$ se colocaron en bloques incompletos; cada familia se colocó en un surco de cinco metros de largo. Cada uno estuvo formado por 10 familias distribuidas aleatoriamente. Las 120 familias de MH y las 110 familias de AH se colocaron en 12 y 11 bloques, respectivamente. En cada bloque incompleto se colocaron como testigos cinco híbridos (Genex 53, Garst 551, D 47, PIONEER 82663 y NK 255), también en forma aleatoria. En la población original NESSC7 $\left(\mathrm{P}_{0}\right)$ las familias correspondientes se sembraron siguiendo la misma distribución de la población NESSC7 $\left(\mathrm{P}_{1}\right)$. En la localidad de Río Bravo el lote experimental fue igual que el de Marín. Cabe mencionar que en cada tipo de familia y en cada localidad se realizaron aleatorizaciones independientes.

Cada familia se consideró como una unidad experimental y en cada una de ellas se estimaron las variables: tamaño de gluma (TG), cobertura de grano (GD) y rendimiento de grano por planta (RG). Para las mediciones se consideró una muestra de 15 granos con gluma de cinco plantas al azar en cada una de las familias, el procedimiento seguido para estimar el tamaño de la gluma y la cobertura de grano fue el mismo utilizado para las líneas exóticas descrito anteriormente; el rendimiento de grano por planta se estimó como el promedio del peso de los granos de las cinco panojas.

Para el análisis de las familias se utilizó el modelo de Diseños Aumentados (Federer 1956, Scott y Milliken 1993) el cuál es el siguiente:

$$
Y_{i j}=\mu+b_{j}+C_{i}+X_{i}\left(C_{i}\right)+\varepsilon_{i j}
$$

Donde: $\mu=$ media de la familia; $b_{j}=$ efecto del bloque, $C_{i}=$ efecto de testigos, $X_{i}\left(C_{i}\right)=$ efecto de familias y $\varepsilon_{i j}=$ error experimental. De este modelo se deriva el siguiente, donde la varianza $\left(\delta^{2}\right)$ se puede expresar como dos efectos: $C_{i} y X\left(C_{i}\right)$ :

$$
Y_{i j}=\mu+b_{j}+\delta^{2}+\varepsilon_{i j}
$$


El análisis de varianza se realizó en cada tipo de familia $(\mathrm{MH}$ y $\mathrm{AH})$ de cada una de las poblaciones NESSC7 $\left(\mathrm{P}_{1}\right)$ y NESSC7 $\left(\mathrm{P}_{0}\right)$ con base en el Statistical Analysis Systems (SAS Institute Inc. 1996).

Con las esperanzas de cuadrados medios se estimaron los componentes de varianza, como se puede observar en el Cuadro 1. Las esperanzas de cuadrados medios de la fuente de variación familias corresponden a la varianza fenotípica de las familias, el VARCOM del SAS separó los componentes del ANOVA en varianza de familias, de bloque y del error experimental y de esta manera se obtuvieron las varianzas genéticas en cada tipo de familia, el error experimental se consideró como la ambiental (Scott y Milliken 1993). Utilizando las familias de $\mathrm{MH}$ se estimó la varianza aditiva mientras que al utilizar las familias de AH se estimó la de dominancia con base en las siguientes fórmulas (Márquez 1985, Hallauer y Miranda 1988):

a) varianza aditiva: $\delta^{2}{ }_{M H}=1 / 4 \delta^{2}$

b) varianza de dominancia: $\delta^{2}{ }_{A H}=\delta^{2}{ }_{A}-1 / 4 \delta^{2}{ }_{D}$

Despejando la varianza aditiva y la varianza de dominancia en cada una de las fórmulas se obtiene:

a) varianza aditiva: $\delta_{A}^{2}=4\left(\delta_{M H}\right)$

b) varianza de dominancia: $\delta_{D}^{2}=4\left(\delta^{2}{ }_{A H}-\delta^{2}{ }_{A}{ }^{*}\right)$

Cuadro 1. Análisis de varianza de las familias de medios hermanos y de autohermanos de la población de sorgo NESSC7 original y con un ciclo de introgresión, evaluados en las localidades Marín, Nuevo León y Río Bravo Tamaulipas, México. Ciclo agrícola primavera 2008.

\begin{tabular}{lcl}
\hline $\begin{array}{l}\text { Fuente de } \\
\text { variación }\end{array}$ & $\begin{array}{c}\text { Componente } \\
\text { de varianza }\end{array}$ & \multicolumn{1}{c}{$\begin{array}{c}\text { Esperanzas de } \\
\text { Cuadrados Medios* }\end{array}$} \\
\hline Testigos & $C i$ & $\delta^{2}{ }_{\mathrm{e}}+0,05 \delta^{2}{ }_{\mathrm{b}}+3 \delta^{2}{ }_{\mathrm{x}(\mathrm{c})}+4,7 \delta^{2}{ }_{\mathrm{c}}$ \\
Familias & $X_{(C i)}$ & $\delta^{2}{ }_{\mathrm{e}}+0,70 \delta^{2}{ }_{\mathrm{b}}+3 \delta^{2}{ }_{\mathrm{x}(\mathrm{c})}$ \\
Bloque & $b j$ & $\delta^{2}{ }_{\mathrm{e}}+4,27 \delta^{2}{ }_{\mathrm{b}}$ \\
Error & $\Sigma_{i j}$ & $\delta^{2}{ }_{\mathrm{e}}$ \\
\hline
\end{tabular}

$* \delta{ }^{2}=$ varianza de bloques, $\delta^{2}{ }_{\mathrm{x}(\mathrm{c})}=$ varianzas de las familias $(\mathrm{MH}$ ó $\mathrm{AH}), \delta^{2}{ }_{\mathrm{c}}=$ varianza de los controles-testigos o checks, $\delta$ ${ }_{\mathrm{e}}^{2}=$ varianza de error, $\mathrm{Ci}=$ efecto de testigos, $X_{(C i)}=$ efecto de las familias, $b j=$ efecto del bloque.
Donde $\delta^{2}{ }_{A}^{*}$ se refiere a la varianza aditiva de las familias de medios hermanos, $\delta_{\mathrm{MH}}=$ varianza de familias de medios hermanos, $\delta^{2}{ }_{A H}=$ varianza de familias de autohermanos, $\delta_{A}^{2}=$ varianza aditiva, $\delta_{D}^{2}=$ varianza de dominancia.

La varianza genética se estimó, como la suma de la varianza aditiva y varianza de dominancia (en este estudio se consideró ausencia de epistásis), la fórmula es $\delta_{G}^{2}=\delta_{A}^{2}+\delta_{D}^{2}$ (Falconer 1975).

Para el caso de la heredabillidad en sentido estrecho $\left(\mathrm{h}^{2}\right)$ se calculó mediante la siguiente fórmula: $h^{2}=$ $\delta^{2}{ }_{A} / \delta_{P}{ }_{P}$ donde $\delta^{2}{ }_{A}=$ varianza aditiva y $\delta_{P}^{2}=$ varianza fenotípica (Falconer 1975).

La varianza fenotípica se obtuvo al sumar la varianza genética y el componente de varianza ambiental (error experimental) y el de bloques (Scott y Milliken 1993).

La respuesta predicha a la selección, para el caso de familias de $\mathrm{MH}$, se estimó con la siguiente fórmula (Falconer 1975, Márquez 1985): $R=i 1 / 4 \delta_{{ }^{2}} / \delta_{P}$; donde $R=$ respuesta predicha a la selección, $\delta^{2}{ }_{A}=$ varianza aditiva y $\delta_{P}=$ desviación estándar fenotípica, $i=$ intensidad de selección (presión de selección del $10 \%)$.

Para la precisión de las estimaciones de la varianza genética se obtuvo el coeficiente de variación genética (Kenapp et al. 1987, Moreno et al. 2002, Bello et al. 2007), la fórmula fue la siguiente $C V G=$ $\sqrt{ } \delta_{{ }_{G}}^{2} /=\mu \times 100$, donde $C V G=$ coeficiente de variación genética, $\sqrt{ } \delta^{2}{ }_{G}=$ desviación estándar genética, = $\mu=$ media del carácter. Los intervalos de confiabilidad para la heredabilidad en sentido estrecho $\left(h^{2}\right)\left(I C-h^{2}\right)$ se utilizó la siguiente fórmula:

$L S=1-\left[(M 1 / M 2) F_{1-\alpha / 2}:{ }_{g l}\right] \mathrm{y}$
$L I=1-\left[(M 1 / M 2) F_{\alpha / 2: g l}\right]^{-1}$,

donde:

LS = límite superior,

$\mathrm{LI}=$ límite inferior,

$\mathrm{M} 1=\mathrm{CM}$ familias,

$\mathrm{M} 2=\mathrm{CM}$ error,

$C M=$ Cuadrado medio, $\alpha=0,05$ de confiabilidad al $95 \%$.

$g l=$ grados de libertad de las familias $\mu$. 


\section{RESULTADOS Y DISCUSIÓN}

En el primer ciclo agrícola se seleccionaron siete líneas de las 14 exóticas que se caracterizaron, éstas presentaron el menor tamaño de gluma y menor cobertura de grano: NAGA-WHITE $(4,2 \mathrm{~mm}$ y $75 \% \mathrm{GD})$, MACIA (4,1 mm y $75 \%$ GD), SC 301 (4,3 mm y $100 \%$ GD), 88 BE2668 (3,7 mm y 75\% GD), SC 27914E (4,8 $\mathrm{mm}$ y $50 \%$ de GD) e IS 4647 (3,6 mm y 61\% GD).

En la localidad de Marín, en ambos tipos de familias se observó un menor tamaño de la gluma en la $\mathrm{P}_{1}$ que en la $\mathrm{P}_{0}$; sin embargo, la mayor disminución se mostró en las familias de $\mathrm{AH}$ donde la longitud disminuyó un $13 \%$, al pasar su tamaño de 4,56 a 3,99 mm (Cuadro 2). La misma tendencia se observó para cobertura de grano, donde para este carácter el incremento de grano descubierto en las familias de $\mathrm{AH}$ fue también de casi el 28\% al incrementarse de 57\% GD a $73 \%$ GD. Para rendimiento de grano por planta los valores medios no cambiaron para los dos tipos de familias entre las dos poblaciones $\left(\mathrm{P}_{1} \mathrm{vs} \mathrm{P}_{0}\right)$.

En la localidad de Río Bravo la tendencia fue exactamente la misma que en la localidad Marín, el tamaño de la gluma disminuyó un $8 \%$ y el porcentaje de grano descubierto se incrementó un $12 \%$ (Cuadro 2).

En las familias de MH la magnitud de los cambios fue menor que en las familias de $\mathrm{AH}$, pero la tendencia fue la misma; el tamaño de la gluma en la $\mathrm{P}_{1}$ en la localidad de Marín disminuyó un $2 \%$ y el porcentaje de grano descubierto se incrementó un $6 \%$, en la localidad de Río Bravo la gluma disminuyó su tamaño casi un $6 \%$ y el porcentaje de grano descubierto se incrementó el $13 \%$.

La disminución en el tamaño de la gluma y la mayor proporción de grano descubierto en la $\mathrm{P}_{1}$ vs $\mathrm{P}_{0}$, confirman el efecto de la introgresión, ya que el uso de las líneas exóticas con menor tamaño de gluma y mayor proporción de grano descubierto, incrementaron la frecuencia génica, con un mayor efecto en la expresión del carácter, generando una población más apta para una cosecha mecanizada con grano más limpio y menores pérdidas de grano.

En las familias de AH se observó el tamaño de la gluma más pequeña $(3,99 \mathrm{~mm})$ y el mayor porcentaje de grano descubierto (73\% grano descubierto), estos datos concuerdan con Marshark (2006) quien menciona que las autofecundaciones en los procesos de formación de las familias de AH tienen mayores efectos en la manifestación del carácter, comparados con las familias de $\mathrm{MH}$.

La introgresión de líneas exóticas con el menor tamaño de gluma y menor cobertura de grano originó una reducción de estas características en la población mejorada.

Cuadro 2. Medias, varianza aditiva, varianza de dominancia, varianza genética y coeficiente de variabilidad genética, para el carácter tamaño de gluma y/o cobertura de grano y rendimiento de grano por planta en la población de sorgo NESSC7 original y con un ciclo de introgresión en el Ciclo agrícola Primavera, en las localidades Marín y Río Bravo, México. 2008.

\begin{tabular}{|c|c|c|c|c|c|c|c|c|c|c|c|c|}
\hline \multirow[b]{2}{*}{ Característica* } & $\mu=-\mathrm{MH}$ & $u=-\mathbf{M H}$ & $\mu=-\mathbf{A H}$ & $\mu=-\mathbf{A H}$ & $\delta^{2}{ }_{A}$ & $\delta_{A}^{2}$ & $\delta_{\mathrm{D}}^{2}$ & $\delta_{D}^{2}$ & $\delta_{G}^{2}$ & $\boldsymbol{\delta}_{{ }_{G}}^{2}$ & CVG & CVG \\
\hline & \multicolumn{12}{|c|}{ Población } \\
\hline Localidad Marín & Po & P1 & P0 & P1 & P0 & P1 & $\mathbf{P 0}$ & $\mathbf{P 1}$ & P0 & P1 & P0 & $\mathbf{P 1}$ \\
\hline Tamaño de gluma & 4,09 & 4,08 & 4,56 & 3,99 & 0,0 & 0,05 & 0,0 & 0,0 & 0,0 & 0,05 & 0,0 & 5,48 \\
\hline Cobertura de grano & 57 & 63 & 57 & 73 & 0,0 & 0,01 & 0,01 & 0,2 & 0,01 & 0,20 & 0,16 & 0,17 \\
\hline $\begin{array}{l}\text { Rendimiento de gra- } \\
\text { no por planta }\end{array}$ & 35 & 33 & 31 & 31 & 509,3 & 5,30 & 0,0 & 0,0 & 509,3 & 5,30 & 64,48 & 6,98 \\
\hline \multicolumn{13}{|l|}{ Localidad Río Bravo } \\
\hline Tamaño de gluma & 4,02 & 3,99 & 4,12 & 3,79 & 0,17 & 0,26 & 0,0 & 0,0 & 0,17 & 0,26 & 10,35 & 12,78 \\
\hline Cobertura de grano & 56 & 69 & 54 & 66 & 0,0 & 0,02 & 0,21 & 0,0 & 0,21 & 0,02 & 0,82 & 0,20 \\
\hline $\begin{array}{l}\text { Rendimiento de gra- } \\
\text { no por planta }\end{array}$ & 35 & 35 & 35 & 34 & 0,0 & 0,0 & 0,00 & 60,10 & 0,0 & 60,10 & 0,0 & 22,15 \\
\hline
\end{tabular}

$* \mu=-\mathrm{MH}=$ media de familias de medios hermanos, $\mu=-\mathrm{AH}=$ media de familias de autohermanos, $\delta^{2}{ }_{\mathrm{A}}=$ varianza aditiva, $\delta^{2}{ }_{\mathrm{D}}=$ varianza de dominancia, $\delta_{G}^{2}=$ varianza genética, $C V G=$ coeficiente de variación genética, $\mathrm{P}_{0}=$ población original, $\mathrm{P}_{1}=$ población mejorada con introgresión. 
El tamaño de gluma más pequeño obtenido en las familias de $\mathrm{AH}(3,99 \mathrm{~mm})$ concuerda con el tamaño de gluma (2,4 a 3,9 $\mathrm{mm}$ ) reportado por el ICRISAT (1999); estos resultados indican que aunque se pudo disminuir el tamaño de la gluma por el proceso de introgresión y considerando el menor tamaño de la gluma de las líneas exóticas utilizadas $(3,56 \mathrm{~mm})$ posiblemente se puede reducir hasta un 33\% para alcanzar los valores bajos que presentaron algunos genotipos de estas líneas.

Para la varianza genética, los efectos de la introgresión también se notaron ya que la $\delta^{2}{ }_{\mathrm{G}}$ fue mayor en la $\mathrm{P}_{1}$ que en la $\mathrm{P}_{0}$ en Marín, N. L., para el tamaño de la gluma el incremento de la $\delta^{2}$ fue un $5 \%$ a favor de $\mathrm{P}_{1}$, mientras que para cobertura de grano fue $20 \%$ (Cuadro 2). La poca $\delta^{2}{ }_{G}$ mostrada en $P_{0}$, demuestra claramente un origen común, donde el uso de otros caracteres como el rendimiento de grano por planta, ha sido el principal criterio de selección. La mayor variabilidad en $\mathrm{P}_{1}$ para los caracteres de interés demuestra, que el haber cruzado nuevas fuentes de éstos hacia la población, sus efectos en cambiar las frecuencias génicas aparecieron en forma muy notoria. Para Río Bravo la tendencia observada fue la misma que en Marín, donde aumentó la $\delta_{G}^{2}$ de 0,17 en $\mathrm{P}_{0}$ a 0,26 en la $\mathrm{P}_{1}$.

La introgresión de genes de las líneas exóticas incrementaron la variabilidad de la población NESSC7 $\left(\mathrm{P}_{1}\right)$ para el caracter tamaño de gluma y/o cobertura de grano, ésto coincide con lo señalado por Menkir et al. (1993) quienes señalaron que la incorporación de líneas exóticas en poblaciones mejoradas a través de la introgresión, incrementa la base genética de los genotipos para efectuar selección y desarrollar líneas puras que contengan el caracter de mejora. Además concuerda con Ron y Hallauer (1997), quienes mencionaron que la introgresión de germoplasma exótico en poblaciones de maíz puede incrementar la variabilidad de un caracter cuantitativo.

En relación a la magnitud y el gradiente observado en las proporciones de la varianza aditiva $\left(\delta^{2}\right)$ en la $\mathrm{P}_{1}$ con respecto a la $\mathrm{P}_{0}$, ocasionada por la introgresión de las líneas exóticas, en la localidad de Marín, se observó una tendencia a incrementar la proporción de la $\delta^{2}{ }_{\mathrm{A}}$ de la $\mathrm{P}_{1}$, en el tamaño de gluma incrementó en $5 \%$ y en la cobertura de grano aumentó $1 \%$; estas proporciones contrastan con las de la $\delta^{2}$ del rendimiento de grano por planta que se redujo de un $99 \%$ en la $\mathrm{P}_{1}$. En la localidad de Río Bravo la magnitud de las proporciones de la $\delta^{2}$ fueron diferentes, en el tamaño de gluma se incrementó un $9 \%$ y la cobertura de grano $2 \%$.
Aunque las magnitudes de las proporciones de la $\delta^{2}{ }_{\mathrm{A}}$ fueron diferentes, la tendencia fue la misma para el tamaño de gluma y la cobertura de grano; el incremento de la $\delta^{2}$ de la $\mathrm{P}_{1}$ posiblemente fue por un efecto aditivo de los genes que al efectuar nuevas combinaciones ocasionadas por el libre apareamiento incrementaron la expresión de la varianza aditiva de este caracter, originando una mayor variabilidad en la $\mathrm{P}_{1}$. Esto coincide con Shell y Becker (1986) quienes mencionaron que el libre apareamiento de las plantas androestériles en una población con el gen $\mathrm{ms}_{3}$ favorecen la recombinación de genes de la población y los de las líneas exóticas introducidas en el primer ciclo de cruzamientos originan cambios en las $\delta^{2}$; ; en el caso de tamaño de gluma y/o cobertura de grano se incrementó la proporción del componente aditivo de la varianza genética (Cuadro 3).

En la $\mathrm{P}_{0}$, el tamaño de gluma y cobertura de grano en la localidad de Marín y el rendimiento de grano por planta en la localidad de Río Bravo, registraron valores negativos de la varianza aditiva en las familias de $\mathrm{MH}$, causado por una mayor expresión de la varianza originada por un alto error experimental. Al respecto Falconer (1975) señaló que la varianza no genética incluye la ambiental y la debida al error experimental y cuando es alta puede encubrir los efectos de la varianza genética (Cuadro 3 ).

En relación a la varianza de dominancia $\left(\delta_{D}^{2}\right)$ para el tamaño de gluma, se consideró inexistente en $\mathrm{P}_{0} \mathrm{y}$ en $\mathrm{P}_{1}$ en las dos localidades, por lo que la expresión de ese caracter se debe a efectos principalmente aditivos de los genes.

No se encontraron otros estudios sobre las varianzas aditivas y de dominancia del caracter tamaño de gluma y/o cobertura de grano, lo que limita establecer términos de comparación.

Considerando las proporciones y las magnitudes de las varianzas fue hacia obtener una mayor proporción de la aditiva para el caracter tamaño de gluma y/o cobertura de grano. En la población con introgresión la proporción de la varianza aditiva para el carácter tamaño de gluma y/o cobertura de grano fue mayor que la proporción de la aditiva en la población original, ésto indica la existencia de variabilidad para este caracter, por lo que es posible hacer selección en sentido favorable para el gen $s g$ (gluma corta) e incrementar los genes favorables que propicien el efecto en la expresión de un tamaño pequeño de gluma y/o menor cobertura de grano y obtener genotipos que muestren una mayor eficiencia en la cosecha del grano de sorgo. 
Cuadro 3. Varianzas genética, fenotípica y ambiental de las poblaciones de sorgo NESSC7 original (P0) y con introgresión (P1), obtenidas del ANOVA, para el tamaño de gluma, cobertura de grano y rendimiento de grano por planta, en las localidades Marín, Nuevo León y Río Bravo en el ciclo agrícola primavera 2008. Tamaulipas, México.

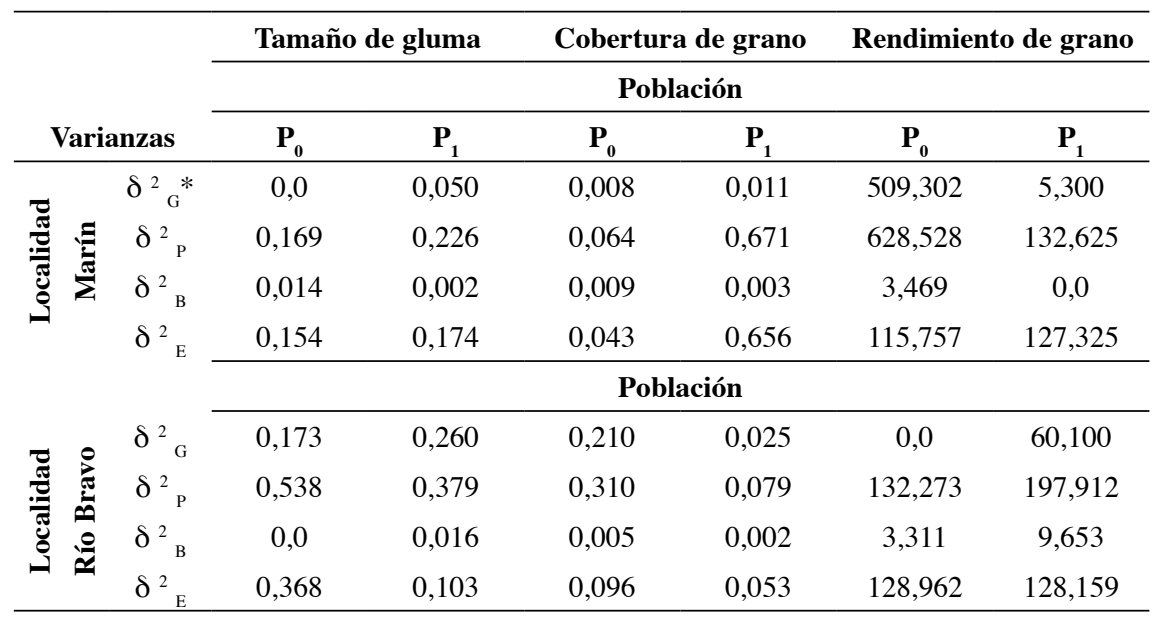

$* \delta{ }^{2}{ }_{\mathrm{G}}$ varianza genética, $\delta{ }_{\mathrm{P}}^{2}=$ varianza fenotípica, $\delta{ }^{2}{ }_{\mathrm{B}}=$ varianza bloques y $\delta^{2}{ }_{\mathrm{E}}=$ varianza error.

Las proporciones de las varianzas genéticas del carácter tamaño de gluma y/o cobertura de grano coincidió con el coeficiente de variabilidad genética, en Marín para el tamaño de gluma en la $\mathrm{P}_{1}$ el CVG fue $5,48 \%$, los demás valores de CVG fueron más altos en la $\mathrm{P}_{1}$ que en la $\mathrm{P}_{0}$. Al respecto, Kenapp et al. (1987), Moreno et al. (2002) y Bello et al. (2007) mencionan que las altas estimaciones de varianza genética generan coeficientes altos indicando alta variabilidad genética del material estudiado.

Los datos obtenidos en este estudio indican que la introgresión de líneas exóticas seleccionadas para tamaño de gluma pequeña y menor cobertura del grano, incrementaron la variabilidad del carácter tamaño de gluma y/o porcentaje de grano descubierto en la población NESSC7 $\left(\mathrm{P}_{1}\right)$ mejorada con un ciclo de introgresión.

La heredabilidad para tamaño de gluma y/o cobertura de grano, la $\mathrm{P}_{1}$ en Marín fue un 22\% superior en la $\mathrm{P}_{0}$, mientras que para cobertura de grano fue de $1 \%$ mayor en la $\mathrm{P}_{1}$ que en la $\mathrm{P}_{0}$. Para la localidad de Río Bravo la $\mathrm{h}^{2}$ para tamaño de gluma fue un $33 \%$ mayor en la $\mathrm{P}_{1}$, mientras que para la cobertura de grano fue de $25 \%$.
Ésta mayor $\mathrm{h}^{2}$ del carácter tamaño de gluma y/o cobertura de grano en la $\mathrm{P}_{1}$ es congruente con las proporciones de la varianza aditiva mostradas en la $\mathrm{P}_{1}$, mientras que la $\mathrm{h}^{2}$ del rendimiento de grano por planta menor en la $\mathrm{P}_{1}$ es congruente con la menor proporción mostrada de la varianza aditiva; no se encontraron estimaciones de heredabilidad para este carácter (Cuadro 4).

En el rendimiento de grano se mostró una mayor heredabilidad en la $\mathrm{P}_{0}$ que en la $\mathrm{P}_{1}$, se encontraron rangos de la $\mathrm{h}^{2}$ en la $\mathrm{P}_{1}$ de 0 a $40 \%$, la mayor $\mathrm{h}^{2}$ es similar a los reportados por Eckbil et al. (1977) quienes estimaron una $\mathrm{h}^{2}$ de 0,38 ; en la $\mathrm{P}_{0}$ los rangos fueron de 0 a $81 \%$, la mayor $\mathrm{h}^{2}$ coincide con lo reportado por Jan-orn et al. (1975), quienes obtuvieron una $\mathrm{h}^{2}$ de 0,82 y los rangos de la $\mathrm{P}_{1}$, similares a los mencionados por Eckbil et al. (1977) quienes estimaron una $\mathrm{h}^{2} \mathrm{de} 0,38$. La h $\mathrm{h}^{2} \mathrm{de}$ cero fue ocasionada por una varianza aditiva debido al componente negativo de las familias de $\mathrm{MH}$.

En la respuesta predicha a la selección $(R)$ para el tamaño de gluma y/o cobertura de grano, se observa una mayor $R$ en la $\mathrm{P}_{1}$ que en la $\mathrm{P}_{0}$, en Marín la proporción del carácter que se pudo obtener al hacer selección a través de familias de $\mathrm{MH}$ fue mayor en $4 \%$ en la $\mathrm{P}_{1}$ que en la 
Cuadro 4. Varianza genética, heredabilidad en sentido estrecho, límites de confianza de la heredabilidad y respuesta predicha a la selección para el carácter tamaño de gluma y/o cobertura de grano y rendimiento de grano por planta en la poblaciones de sorgo NESSC7 en el ciclo agrícola primavera 2008, en las localidades Marín y Río Bravo. México.

\begin{tabular}{lcccccccc}
\hline & \multicolumn{7}{c}{ Población } \\
\cline { 2 - 8 } & $\mathbf{P}_{\mathbf{0}}{ }^{*}$ & $\mathbf{P}_{\mathbf{1}}$ & $\mathbf{P}_{\mathbf{0}}$ & $\mathbf{P}_{\mathbf{1}}$ & & $\mathbf{P}_{\mathbf{1}}$ & $\mathbf{P}_{\mathbf{0}}$ & $\mathbf{P}_{\mathbf{1}}$ \\
\cline { 2 - 9 } Característica & $\boldsymbol{\delta}_{\mathbf{G}_{\mathbf{G}}}$ & $\boldsymbol{\delta}_{\mathbf{G}}{ }_{\mathbf{G}}$ & $\mathbf{h}^{\mathbf{2}}$ & $\mathbf{h}^{\mathbf{2}}$ & & $\mathbf{L . C}$ & $\mathbf{R}$ & $\mathbf{R}$ \\
\hline Localidad Marín & & & & & & & & \\
Tamaño de gluma & 0,00 & 0,05 & 0,00 & 0,22 & \pm & 4,56 & 0,00 & 0,04 \\
Cobertura de grano & 0,01 & 0,20 & 0,00 & 0,01 & \pm & 1,72 & 0,00 & 0,003 \\
Rendimiento de grano por planta & 509,3 & 5,30 & 0,81 & 0,04 & \pm & 0,002 & 4,45 & 0,11 \\
Localidad Río Bravo & & & & & & & & \\
Tamaño de gluma & 0,17 & 0,26 & 0,02 & 0,69 & \pm & 2,7 & 0,01 & 0,16 \\
Cobertura de grano & 0,21 & 0,02 & 0,0 & 0,25 & \pm & 1,54 & 0,00 & 0,03 \\
Rendimiento de grano por planta & 0,0 & 60,1 & 0,0 & 0,0 & \pm & 0,001 & 0,0 & 0,0 \\
\hline
\end{tabular}

$* \delta_{\mathrm{G}}^{2}=$ varianza genética, L. C. = límites de confianza de la heredabilidad, R=respuesta predicha a la selección, $\mathrm{P}_{0}=$ población original, $\mathrm{P}_{1}=$ población mejorada con introgresión, $\mathrm{h}^{2}=$ heredabilidad.

$\mathrm{P}_{0} \mathrm{y}$ para la cobertura de grano la proporción es mayor en la $\mathrm{P}_{1}$ en $0,3 \%$. Algo similar ocurrió en Río Bravo, la proporción de la $R$ fue mayor en $15 \%$ y para cobertura de grano la $R$ fue mayor en $3 \%$ en la $\mathrm{P}_{1}$. No se encontraron otros estudios sobre la $R$ en este carácter.

La $R$ para rendimiento de grano fue menor en la $\mathrm{P}_{1}$ que en la $\mathrm{P}_{0}$, en la localidad Marín la $R$ disminuyó $97,5 \%$ en la $\mathrm{P}_{1}$ y en la localidad Río Bravo la heredabilidad fue cero en la $\mathrm{P}_{1}$ y $\mathrm{P}_{0}$ (ocasionada por el componente negativo) originó un $R$ de cero, esto indica que en esta población ya se había seleccionado para rendimiento de grano y este carácter no se consideró en la introgresión.

Las proporciones de la respuesta predicha a la selección, la varianza genética y la heredabilidad en sentido estrecho mostraron concordancia con la varianza aditiva, además, los CVG y los límites de confiabilidad de la heredabilidad indican que se obtuvo precisión en las estimaciones.

El carácter tamaño de gluma y/o cobertura de grano en la población NESSC7 $\left(\mathrm{P}_{1}\right)$ presentó mayor proporción de varianza aditiva, mayor heredabilidad en sentido estrecho y mayor respuesta a la selección y siendo estas proporciones mayores en la $\mathrm{P}_{1}$ vs $\mathrm{P}_{0}$ por efecto de la introgresión. Con base en los valores de la $\delta^{2}{ }_{\mathrm{A}}$ y $\delta^{2}{ }_{\mathrm{D}}$ se puede especular que la selección recurrente es el método a seguir y permite utilizar el doble de la varianza aditiva contenida en la población (Márquez 1988).

\section{LITERATURA CITADA}

Bello, D; Obilana, AT. 1985. Inheritance studies in grain sorghum. Sorghum Newsletter. Nigeria. p. 28-76.

Bello, D; Kadams, AM; Simon, SY; Mashi, DS. 2007. Studies on genetic variability in cultivated sorghum (Sorghum bicolor L. Moench) cultivars of Adamawa State Nigeria. American-Euriasian Journal Agricultural \& Enviroment Science 2(3):297-302.

Clayton, WD; Renvoize, SA. 1986. Genera graminum grasses of the world. In: Kew Bulletin Addition Series XIII. London. Editorial: Royal Botanic Gardens. 389 p.

Chaparro, CJM. 1997. Parámetros de operación en las combinadas. Cosechadoras de cereales. Universidad de Colombia, Colombia (en línea). Consultado 10 ene. 2006. Disponible en http://www. infoagro.com

Dogget, H. 1970. Sorghum. Wiley \& Sons. London: Longman. New York, USA. 403 p. 
Eckebil, JP; Ross, WN; Gardner, CO; Maranville, JW. 1977. Heritability estimates genetic correlation and predicted gains from $S_{1}$ progeny test in three grain sorghum random-mating populations.ARS, USDA, Nebraska, E.U.A. Crops Science 17:373-377.

Everety, J. 2000. Molecular and morphological evolution in the Andropogonae. Grass: Systematics and Evolution. Editorial CSIRO. Australia. 402 p.

Falconer, DS. 1975. Introducción a la genética cuantitativa. Editorial Continental. Traductor: Fidel Márquez Sánchez. 5 ed. México, DF. 430 p.

Federer, WT. 1956. Augmented (or hoonuiaku) designs. Hawaii Plant Records 2:191-208.

Hallauer, AR; Miranda, JB. 1988. Quantitative genetics in maize breeding. Ioawa State University. USA. 468 p.

House, RL. 1985. A guide to sorghum breeding. Patancheru, India: International Crops Research Institute for the Semi-Arid Tropics ICRISAT. 2 ed. India. 206 p.

ICRISAT (International Crops Research Institute for the Semi-Arid Tropics). 1999. Instituto Internacional de los Recursos Genéticos de la Planta. International Crops Research Institute for the Semi-Arid Tropics (ICRISAT), Italia (en línea). Consultado 22 sept. 2005. Disponible en www.icrista.org/Newsletter/

Jan-orn, J; Gardner, CO; Ross, WM. 1975. Quantitative genetics studies of the NP3R random-mating grain sorghum population. Crops Science 16:489-496.

Kenapp, SJ; Ross, W; Strpou, W. 1987. Precision of genetic variance and heritability estimates from sorghum populations. Crop Science 27:265-268.

Kenga, R; Tenkouano, A; Gupta, SC; Alabi, SO. 2006. Genetic and phenotypic association between yield components in hybrid sorghum (Sorghum bicolor L. Moench) populations. Eupytica 150 (3):319-326.

Khusnetdinova, TG; El'konin, AL. 1989. Inteheritance of some qualitative characters in sorghum. Kompleksnye issledovaniya po selektsii sorghum. Zenograd. USSR. p. 79-93

Márquez S. F. 1985. Genotecnia vegetal. Tomo I. Editorial. AGT. México, DF. 357 p.

Márquez S. F. 1988. Genotecnia vegetal. Tomo II. Editorial AGT. México, DF. 657 p.
Márquez, SF; Sahagún, JC. 1994. Estimation of genetic variance with maternal half-sib families. Mayadica. 39:197-201.

Márquez, MF. 1997. Estimación de varianzas genéticas usando líneas autofecundadas como progenitores en maíz. Agrociencias 31:171-175.

Mashark, SA. 2006. Genotypic variation of Sorghum bicolor (L.) Moench in response to different cropping systems. Agonomy Journal 5(4):634-640.

Menkir, A; Bramer-Cox, PJ; Witt, MD. 1993. Comparisons of methods for introgressing exotic germplasm into adapted sorghum. Theoretical and Applied Genetics. 89:1432-2242.

Moreno, MM; Peña L, A; Sahágun, J; Rodríguez, JE; Mora, R. 2002. Varianza aditiva, heredabilidad y correlaciones en la variedad M1-Fitotécnia de tomate de cáscara (Physalis ixocarpa Brot). Revista Fitotecnia Méxicana 25(3):231-237.

Ron, J; Hallauer, AR. 1997. Utilization of exotic maize germplasm. Plant Breeding 14:165-187.

SAS Institute.1996.Statistical Analysis System User's Guide. SAS Institute.Cary. USA. 956 p.

Secretaria de Comercio y Fomento Industrial. 1994. Norma Oficial Mexicana NMX-FF-037-1994. Productos alimenticios no industrializados, alimentos para uso humano-cereales-sorgo (Sorghum vulgare L.).Especificaciones y métodos de prueba. (en línea). Dirección General de normas. 10 p. Consultado 24 mayo 2006. Disponible en http://www.sagarpa.gob. $\mathrm{mx} /$ agricultura/info

Shell, FW; Becker, HC. 1986. Yield and yield stability in a balance system of widely differing population structures. In: Zea mays L. Plant Bredding 97:30-31.

Scott, RA; Milliken, GA. 1993. A SAS program for analyzing augmented randomized complete block designs. Crops Science 33:865-867.

USDA-ARS (United States Department of Agriculture - Agricultural Research Service). 2003. Germplasm Resources Information Network (GRIN). National Genetic Resources Program. National Germplasm Resources Laboratory (en línea). Beltsville, Maryland. Consultado 8 feb. 2009. Disponible en http://www.ars-grin.gov/cgi-bin/npgs/html 